\section{Commentary: Does a meta-analysis of controversial trials yield controversial results?}

\author{
Derrick Y. Tam, MD, and \\ Stephen E. Fremes, MD, MSc
}

The optimal revascularization strategy for unprotected left main (LM) coronary artery disease (CAD) remains highly controversial since the most recent publication of the Evaluation of Xience versus Coronary Artery Bypass Surgery for Effectiveness of Left Main Revascularization (EXCEL) trial. ${ }^{1,2}$ Heated arguments have erupted at scientific meetings and social media regarding the correct interpretation of the findings of the EXCEL trial, specifically with regard to the appropriate definition of periprocedural myocardial infarction (MI). ${ }^{2}$ Whereas the most recent edition of the European revascularization guidelines recommend percutaneous coronary intervention (PCI) or coronary artery bypass grafting (CABG) for LM disease in patients with a low Synergy Between Percutaneous Coronary Intervention with Taxus and Cardiac Surgery (SYNTAX) score (class Ia), the European Association for Cardio-Thoracic Surgery has formally withdrawn support for these current treatment recommendations. ${ }^{3}$ Against this backdrop, it becomes critically important to look at the totality of evidence when it comes to revascularization strategies for left main CAD.

Gallo and colleagues ${ }^{4}$ tackle this divisive issue in a metaanalysis of randomized controlled trials with at least 5 years of follow-up data comparing PCI to CABG for unprotected LM CAD. Overall, 5 studies were included: EXCEL, the

\footnotetext{
From the Division of Cardiac Surgery, Schulich Heart Centre, and Division of Cardiac Surgery, Department of Surgery, Sunnybrook Health Sciences Centre, University of Toronto, Toronto, Ontario, Canada.

Disclosures: The authors reported no conflicts of interest.

The Journal policy requires editors and reviewers to disclose conflicts of interest and to decline handling or reviewing manuscripts for which they may have a conflict of interest. The editors and reviewers of this article have no conflicts of interest.

Received for publication April 11, 2020; revisions received April 11, 2020; accepted for publication April 12, 2020; available ahead of print April 23, 2020.

Address for reprints: Stephen E. Fremes, MSc, MD, FRCSC, FACP, FACC, Division of Cardiac Surgery, Schulich Heart Centre, and Division of Cardiac Surgery, Department of Surgery, Sunnybrook Health Sciences Centre, University of Toronto, 2075 Bayview Ave, Room H4 05, Toronto, Ontario M4N 3M5, Canada (E-mail: stephen.fremes@sunnybrook.ca).

J Thorac Cardiovasc Surg 2022;163:106-8

0022-5223/\$36.00

Copyright (c) 2020 by The American Association for Thoracic Surgery

https://doi.org/10.1016/j.jtcvs.2020.04.052
}

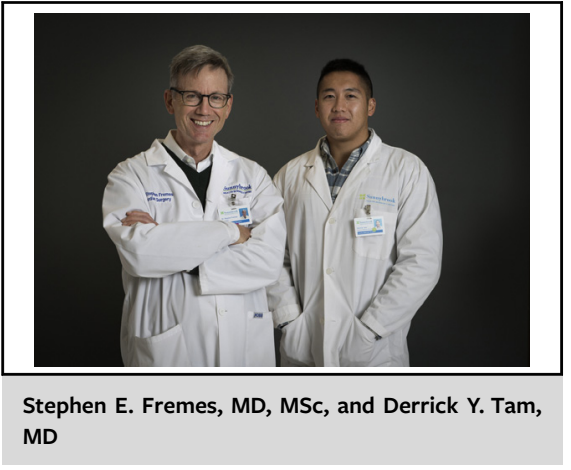

CENTRAL MESSAGE

Coronary artery bypass grafting remains a safe and effective treatment option in patients with left main coronary artery disease.

Nordic-Baltic-British left main revascularization study, Ten-Year Outcomes of Premier of Randomized Comparison of Sirolimus-Eluting Stent Implantation versus Coronary Artery Bypass Surgery for Unprotected Left Main Coronary Artery Stenosis (PRECOMBAT), SYNTAX-LM, and a study by Boudriot and colleagues. There were several important findings. Early outcomes were similar between CABG and PCI with the exception of stroke, which favored PCI. However, stroke was similar between the 2 arms at 5 years. At 5 years, both new MI and need for repeat revascularization favored $\mathrm{CABG}$ although there was no difference in long-term mortality. A recently published meta-analysis also examined these 5 randomized controlled trials, the additional information from this analysis includes early (30 days), midterm (1 year), and late outcomes (5 years). ${ }^{4,5}$ Overall, the findings are consistent and suggest that there may be an upfront higher risk with CABG in the short term that is mitigated over time and that CABG provides durable results with fewer future MIs and repeat revascularization procedures.

There are important implications to these findings. In patients who are good surgical candidates, CABG should be the preferred approach because it is associated with less major cardiac and cerebrovascular events (MACCE) at 5 years. Some have criticized that repeat revascularization and MI have driven this difference in MACCE and that perhaps these are acceptable trade-offs for a less invasive approach from a patient's perspective, yet recent post hoc analysis of the EXCEL trial suggests that the need for repeat revascularization is associated with a doubling of mortality at 
3 years. ${ }^{6}$ The influence of incomplete revascularization on worse late MACCE events and mortality is well known, ${ }^{7}$ and given the large number of patients in these trials with more than isolated LM disease (70\%-90\%), we should not be surprised by the higher number of late MIs and repeat revascularizations in the PCI group. The association of increased late MI with PCI compared with CABG is likely explained by the differing mechanism of how these techniques revascularize diseased vessels. Surgical revascularization offers potential protection of the distal coronary bed such that should native disease progress CABG grafts serve as a form of collateralization, which is not the case with PCI. ${ }^{8}$ PCI should be reserved for those at high surgical risk or those at high risk for periprocedural stroke-a patient-level meta-analysis of 11 CABG versus PCI trials by Head and colleagues ${ }^{9}$ demonstrated that postprocedure stroke was higher within the first 30 days of the procedure $(1.1 \%$ vs $0.4 \%$ for CABG and PCI, respectively) but similar between 31 days and 5 years $(2.4 \%$ vs $2.1 \%$; hazard ratio, $1.05 ; 95 \%$ confidence interval, $0.80-1.38$ ).

Nonetheless, there are important limitations that must be considered in interpreting these findings. Although the inclusion of patients from randomized controlled trials helps reduce confounding due to treatment allocation bias, this limits generalizability to patient population that was male dominated $(75 \%)$, low-intermediate SYNTAX score (2130 ), and a mean age around 65 years (62-68 years). In other words, these were highly selected patients and indeed, a close examination of the EXCEL trial registry of 1000 patients who were ineligible for randomization suggests that $45 \%$ of patients were excluded based on anatomy that was not favorable to PCI and $18 \%$ of patients were excluded due to unfavorable anatomic characteristics for CABG. ${ }^{10}$ We also note that the trial study periods for SYNTAX, PRECOMBAT, and the study by Boudriot and colleagues started in the early 2000s and Nordic-BalticBritish left main revascularization study and EXCEL started in the late 2000s, with the former 3 trials completed before 2010. There have been technical advances for both CABG and PCI since the start of the early trials. In CABG, there has been a move toward increased use of arterial conduits to improve graft patency while for PCI later generation stent platforms and novel techniques have developed to treat more complex LM disease. ${ }^{11}$ In terms of methodology, although study-level meta-analyses and individual-patient-level meta-analyses may produce similar point estimates, the use of individual-patient-level data allows authors to examine particular subgroups and facilitate the inclusion of time-to-event data rather than just treating late outcomes as binary events. ${ }^{12-14}$ Finally, whereas follow-up of most studies was limited to 5 years, longer follow-up may help elucidate differences in mortality outcomes between CABG and PCI. That said, recent publication of 10-year results for the SYNTAX trial extended follow-up ${ }^{15}$ and the presentation of results of the older PRECOMBAT trial at the American College of Cardiology 2020 meeting suggested no difference for late mortality between CABG and PCI at 10 years. ${ }^{16}$

Despite these limitations, Gallo and colleagues ${ }^{4}$ must be congratulated for conducting this important meta-analysis comparing $\mathrm{PCI}$ to $\mathrm{CABG}$ in $\mathrm{LM}$ disease. Given that either $\mathrm{PCI}$ or CABG is feasible in many patients, discussion within heart teams is strongly recommended to individualize treatment decisions regarding patient characteristics and anatomic factors. CABG remains a safe and durable procedure and should be the preferred option in those with complex anatomy and those at low-to-intermediate surgical risk. Controversy may surround some of these clinical trials, but the findings from this meta-analysis are as expected and continue to support the role of CABG in LM disease.

\section{References}

1. Stone GW, Kappetein AP, Sabik JF, Pocock SJ, Morice MC, Puskas J, et al. Fiveyear outcomes after PCI or CABG for left main coronary disease. $N$ Engl J Med. 2019;381:1820-30

2. Bjørnstad JL, Bendz B. Periprocedural myocardial injury in the EXCEL trial. Eur Heart J. 2020;40:1930

3. Neumann F-J, Sousa Uva M, Ahlsson A, Alfonso F, Banning AP, Benedetto U, et al. 2018 ESC/EACTS guidelines on myocardial revascularization. Eur Hear J. 2019;40:87-165

4. Gallo M, Blitzer D, Laforgia PL, Doulamis IP, Perrin N, Bortolussi G, et al. Percutaneous coronary intervention versus coronary artery bypass graft for left main coronary artery disease: a meta-analysis. J Thorac Cardiovasc Surg. 2022;163:94-105.e15.

5. Ahmad Y, Howard JP, Arnold AD, Cook CM, Prasad M, Ali ZA, et al. Mortality after drug-eluting stents vs. coronary artery bypass grafting for left main coronary artery disease: a meta-analysis of randomized controlled trials. Eur Heart J. 2020;60:e44.

6. Giustino G, Serruys PW, Sabik JF III, Mehran R, Maehara A, Puskas JD, et al. Mortality after repeat revascularization following PCI or CABG for left main disease: the EXCEL trial. JACC Cardiovasc Interv. 2020;13:375-87.

7. Takagi H, Watanabe T, Mizuno Y, Kawai N, Umemoto T, ALICE (All-Literature Investigation of Cardiovascular Evidence) Group. A meta-analysis of adjusted risk estimates for survival from observational studies of complete versus incomplete revascularization in patients with multivessel disease undergoing coronary artery bypass grafting. Interact Cardiovasc Thorac Surg. 2014 18:679-82.

8. Doenst T, Haverich A, Serruys P, Bonow RO, Kappetein P, Falk V, et al. PCI and CABG for treating stable coronary artery disease: JACC review topic of the week J Am Coll Cardiol. 2019;73:964-76.

9. Head SJ, Milojevic M, Daemen J, Ahn JM, Boersma E, Christiansen EH, et al. Stroke rates following surgical versus percutaneous coronary revascularization. J Am Coll Cardiol. 2018;72:386-98.

10. Stone GW, Sabik JF, Serruys PW, Simonton CA, Généreux P, Puskas J, et al. Everolimus-eluting stents or bypass surgery for left main coronary artery disease. N Engl J Med. 2016;375:2223-35.

11. Tam DY, Bakaeen F, Feldman DN, Kolh P, Lanza GA, Ruel M, et al. Modality selection for the revascularization of left main disease. Can J Cardiol. 2019; 35:983-92.

12. Williamson PR, Smith CT, Hutton JL, Marson AG. Aggregate data meta-analysis with time-to-event outcomes. Stat Med. 2002;21:3337-51.

13. Gaudino M, Benedetto U, Fremes S, Biondi-Zoccai G, Sedrakyan A, Puskas JD, et al. Radial-artery or saphenous-vein grafts in coronary-artery bypass surgery. $N$ Engl J Med. 2018;378:2069-77.

14. Gaudino M, Rahouma M, Abouarab A, Leonard J, Kamel M, Di Franco A, et al. Radial artery versus saphenous vein as the second conduit for coronary artery bypass surgery: a meta-analysis. J Thorac Cardiovasc Surg. 2019;157: 1819-25.e10. 
15. Thuijs DJFM, Kappetein AP, Serruys PW, Mohr FW, Morice MC, Mack MJ, et al. Percutaneous coronary intervention versus coronary artery bypass grafting in patients with three-vessel or left main coronary artery disease: 10-year follow-up of the multicentre randomised controlled SYNTAX trial. Lancet. 2019;394: $1325-34$.
16. Seung-Jung Park S-J. Ten-year outcomes After drug-eluting stents versus coronary artery bypass grafting for left main coronary disease: extended follow-up of the PRECOMBAT trial. Presented at: American College of Cardiology Virtual Annual Scientific Session Together with World Congress of Cardiology; March 30, 2020; Chicago, IL.
See Article page 94

\section{Commentary: The left main controversy: Is this a real subgroup requiring custom clinical recommendations?}

\author{
Mario Gaudino, MD, ${ }^{\mathrm{a}}$ Domenico Pagano, $\mathrm{MD},{ }^{\mathrm{b}}$ and \\ Nick Freemantle, $\mathrm{PhD}^{\mathrm{c}}$
}

For 3 decades, coronary artery bypass grafting (CABG) has been the standard of care for patients with left main coronary artery stenosis (LMCAS), a practice based on trials that showed improved survival compared with medical management. ${ }^{1}$ The Synergy Between Percutaneous Coronary Intervention with Taxus and Cardiac Surgery (SYNTAX) trial was the first large trial to compare percutaneous coronary intervention (PCI) with CABG in the treatment of coronary artery disease. ${ }^{2}$ The SYNTAX protocol included a prespecified exploratory analysis in the subgroup of patients with LMCAS that was underpowered for the primary composite end point of all-cause mortality, stroke,

From the ${ }^{\mathrm{a}}$ Department of Cardiothoracic Surgery, Weill Cornell Medicine, New York, NY; ${ }^{b}$ Department of Cardiothoracic Surgery, University Hospital Birmingham, University of Birmingham, Birmingham, United Kingdom; and ${ }^{\mathrm{c}}$ Institute of Clinical Trials and Methodology, University College London, London, United Kingdom.

Disclosures: Dr Pagano is secretary general of the European Association for CardioThoracic Surgery, and International Director of the Society of Thoracic Surgeons. Dr Freemantle has received funding for consulting from sanofi-Aventis, MSD, AstraZeneca, Takeda, Novo Nordisk, Ipsen, Allergan, and PCT. His organization receives funding from the European Association for Cardio-Thoracic Surgery for methodological and educational work. Dr Gaudino reported no conflicts of interest.

The Journal policy requires editors and reviewers to disclose conflicts of interest and to decline handling or reviewing manuscripts for which they may have a conflict of interest. The editors and reviewers of this article have no conflicts of interest.

Received for publication April 14, 2020; revisions received April 14, 2020; accepted for publication April 15, 2020; available ahead of print May 6, 2020.

Address for reprints: Mario Gaudino, MD, Department of Cardiothoracic Surgery, Weill Cornell Medicine, 525 E 68th St, New York, NY 10065 (E-mail: mfg9004@med.cornell.edu).

J Thorac Cardiovasc Surg 2022;163:108-10

$0022-5223 / \$ 36.00$

Copyright (c) 2020 by The American Association for Thoracic Surgery

https://doi.org/10.1016/j.jtcvs.2020.04.102

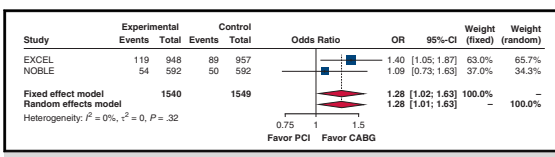

Meta-analytic estimates for all-cause mortality pooling the EXCEL and NOBLE trials.

CENTRAL MESSAGE
Left main disease does not
describe a defined biological or
statistically discrete subgroup of
patients in available studies; clin-
ical recommendations on multi-
vessel disease should apply to
these patients.

myocardial infarction, and repeat revascularization. This subgroup comparison was null at 5 years and generated the hypothesis that, in patients with low and medium coronary disease complexity, the 2 treatments may achieve similar outcomes. ${ }^{3}$ This hypothesis was tested in the Evaluation of XIENCE versus Coronary Artery Bypass Surgery for Effectiveness of Left Main Revascularization (EXCEL) and Nordic-Baltic-British Left Main Revascularisation (NOBLE) trials. ${ }^{4,5}$ The 2 trials used different primary composite end points ${ }^{6}$ and found qualitatively different results. In brief, EXCEL reported no statistically significant difference between CABG and PCI at 5-year follow-up in the primary composite outcome of death from any cause, stroke, or myocardial infarction (including perioperative and spontaneous events). All-cause mortality curves diverged in favor of $\mathrm{CABG}$ and the difference was nominally significant at 5-year follow-up. ${ }^{7}$ NOBLE reported a significant advantage of CABG for the primary composite end point of allcause mortality, nonprocedural myocardial infarction, repeat revascularization, and stroke at 5 years, without significant difference in all-cause mortality (although the event rate was low and the confidence interval $[\mathrm{CI}]$ wide) ${ }^{8}$ 